\title{
Effect of neoadjuvant chemotherapy in patients with triple-negative breast cancer: A meta-analysis
}

\author{
MUYOU TIAN $^{1,2}$, YAHUA ZHONG ${ }^{1,2}$, FUXIANG ZHOU $^{1,2}$, CONGHUA XIE $^{1,2}$, \\ YUNFENG ZHOU ${ }^{1,2}$ and ZHENGKAI LIAO ${ }^{1,2}$ \\ ${ }^{1}$ Hubei Cancer Clinical Study Center, Hubei Key Laboratory of Tumor Biological Behaviors; \\ ${ }^{2}$ Department of Radiation Oncology and Medical Oncology, Zhongnan Hospital, Wuhan University, \\ Wuhan, Hubei 430071, P.R. China
}

Received June 26, 2014; Accepted March 11, 2015

DOI: $10.3892 / 01.2015 .3072$

\begin{abstract}
The present meta-analysis aimed to evaluate the effect of neoadjuvant chemotherapy on pathological complete response ( $\mathrm{pCR}$ ) and survival rate in patients with triple-negative breast cancer (TNBC). Specific inclusion and exclusion criteria were used to conduct a search of the available databases, in order to find studies performed between January 2006 and January 2014. The bibliographies of the included studies were examined with the same criteria. The Grading of Recommendations Assessment, Development and Evaluation (GRADE) working group framework was used to evaluate the included studies, and RevMan 5.1 and GRADEprofiler 3.6 were used to analyze the extracted data. A total of 19 studies with 6,180 patients were included. The meta-analysis revealed that the pCR rates in patients with TNBC were significantly higher than those in patients with non-TNBC. The 5-year disease-free survival (DFS) and overall survival (OS) rates were significantly lower in the patients with TNBC compared with those with non-TNBC. Furthermore, these survival rates were significantly higher in the patients with TNBC who achieved a pCR compared with those in the patients who did not achieve a pCR. pCR rates were higher among the patients with TNBC with high Ki-67 expression than among those with low Ki-67 expression. The patients with TNBC exhibited lower survival rates compared with those with non-TNBC, but achieved higher pCR rates. Moreover, those patients achieving a pCR exhibited improved 5 -year survival rates, suggesting that the pCR rate could be predictive of survival in patients with TNBC. In addition, high $\mathrm{Ki}-67$ expression may predict the likelihood of a pCR. However, future multicenter randomized controlled trials are required to enhance the quantity and quality of the clinical evidence.
\end{abstract}

Correspondence to: Dr Zhengkai Liao, Department of Radiation Oncology and Medical Oncology, Zhongnan Hospital, Wuhan University, 169 Donghu Road, Wuhan, Hubei 430071, P.R. China E-mail: zliao@whu.edu.cn

Key words: triple-negative breast cancer, neoadjuvant chemotherapy, pathological complete response, survival, molecular marker

\section{Introduction}

Breast cancer is subdivided into five types, namely luminal A, luminal B, human epidermal growth factor receptor-2 (HER-2)-positive, normal-like and basal-like breast cancer, according to cellular-molecular phenotype (1). In total, $90 \%$ of triple-negative breast cancer (TNBC) cases are classified as basal-like. TNBC, as its name suggests, has an estrogen receptor (ER)-negative, progesterone receptor (PR)-negative and HER-2-negative phenotype, and accounts for $15 \%$ of all breast cancer cases. TNBC is highly aggressive, with a high propensity for metastasis and a poor survival rate $(2,3)$. Therefore, endocrine and molecularly targeted therapies are unsuitable for patients with TNBC, and chemotherapy is the only systemic therapy available.

Neoadjuvant chemotherapy (NAC) has become a widely applied treatment for early-stage breast cancer (4). NAC can downstage a tumor, potentially enabling breast-conserving surgery for patients who may have otherwise required mastectomy $(5,6)$. Based on preclinical studies in animal models, it was hypothesized that NAC may diminish the micrometastases of breast cancer (7). In general, tumor size and lymph node number are the foremost prognostic predictors of solid tumors following systemic therapy, but they are not appropriate for determining the response to NAC. A pathological complete response (pCR) is used as a short-term evaluation index for the efficacy of NAC.

Patients with TNBC usually achieve a higher pCR rate $(8,9)$. Furthermore, it has been reported that patients with TNBC and those with non-TNBC who achieved pCR following NAC have similar long-term survival rates. By contrast, the 5-year disease-free survival (DFS) rates of patients who did not achieve pCR following NAC differed significantly between those patients with TNBC and those with non-TNBC (10). However, a meta-analysis in 2011 reported that $\mathrm{pCR}$ is an independent prognostic factor of overall survival (OS), DFS and relapse-free survival (RFS) for patients with TNBC (11). Other studies indicated that molecular biomarkers, including Ki-67 antigen, tumor suppressor p53, epidermal growth factor receptor (EGFR), and cytokeratin $(\mathrm{CK}) 5$ and 6 , may predict the $\mathrm{pCR}$ rate of patients with TNBC following NAC $(12,13)$. 
In this meta-analysis, data was extracted and the short-term efficacy (pCR) and long-term survival (DFS and OS) rates of patients with TNBC treated with NAC were analyzed. In order to provide prognostic guidance for TNBC patients, the present meta-analysis attempted to further prove the association between pCR and long-term survival, and to determine if any biomarkers were predictive of the $\mathrm{pCR}$ rate.

\section{Materials and methods}

Inclusion and exclusion criteria. Prospective or retrospective controlled trials were included, regardless of the allocation, concealment or blinding. All the following criteria had to be met for inclusion in the meta-analysis: i) NAC must have been the primary initial therapy; ii) patients must have had stage I-III breast cancer; iii) immunohistochemical staining should have confirmed hormone receptor status and/or fluorescence in situ hybridization should have confirmed HER-2 status; and iv) the pCR rate, and DFS or OS rates had to have been reported. Studies were excluded if they met any of the following criteria: i) Repetitive publication; ii) small sample size; iii) abstract only; and iv) no sufficient raw data and data unavailable on request.

Literature search strategy. TNBC is a concept that was initially introduced in 2006 (14); therefore, searches of the PubMed database, the China Knowledge Resource Integrated Database, the China Science and Technology Journal Database, and the WanFang database were performed using date limits of between January 2006 and January 2014. Papers in the Chinese and English languages were searched. Retrieval keywords included i) triple-negative breast cancer or TNBC; ii) neoadjuvant chemotherapy or NAC; iii) pathological complete response or pCR; iv) survival or disease-free survival or DFS or overall survival or OS; v) molecular marker or CK5/6 or p53 or Ki-67; and vi) combinations of these terms, including i)+ii), i)+ii)+iii), i)+ii)+iv) and i)+ii)+v).

Data extraction. Based on the aforementioned strategies, studies were selected and their eligibility was confirmed by three independent researchers. The following information was extracted from each study: Authors' names, year of publication, study type, total number of patients, median patient age, primary tumor-node-metastasis (TNM) stage, NAC regimen and survival data.

Quality evaluation. The Grading of Recommendations Assessment, Development and Evaluation (GRADE) working group framework was used to evaluate the collated data; accordingly, high, medium, low or very low grades were awarded with regard to quality. Randomized controlled trials were considered to be of a high grade, but the following factors were also considered: Risk of bias, indirectness, inconsistency, imprecision and publication bias. Case-control and cohort studies were considered to be of a medium grade.

Statistical analyses. Review Manager software (RevMan, version 5.1 for Windows; The Cochrane Collaboration, Oxford, UK) was used to conduct the meta-analysis. Odds ratio $(\mathrm{OR})$ and $95 \%$ confidence interval $(95 \% \mathrm{CI})$ values were calculated. A $\chi^{2}$ test was used to evaluate heterogeneity in the

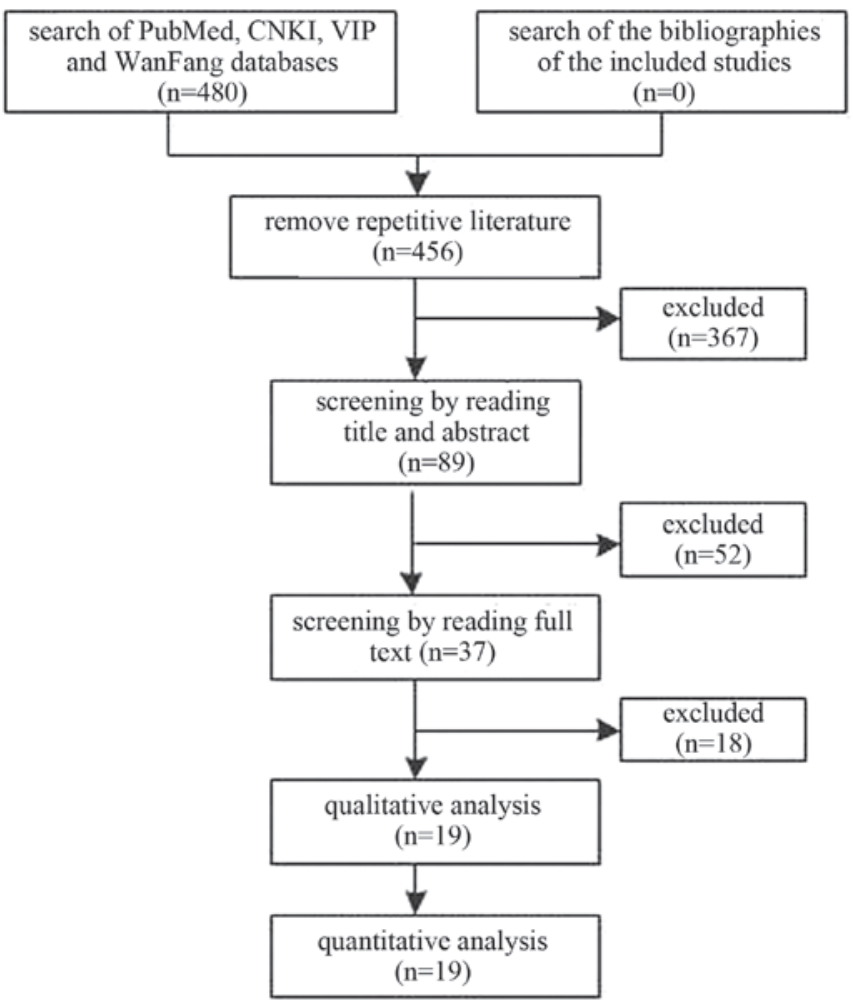

Figure 1. Flow-chart of the literature search process. CNKI, China Knowledge Resource Integrated database; VIP, China Science and Technology Journal database.

data. The fixed-effects model was used for studies without significant heterogeneity $\left(\mathrm{I}^{2} \leq 50 \%\right.$ or $\left.\mathrm{P} \geq 0.10\right)$, whereas the random-effects model was used for studies with significant heterogeneity. Funnel plots were generated using RevMan to detect publication bias. GRADEpro software (version 3.6 for Windows; The Cochrane Collaboration) was used to conduct the quality evaluation.

\section{Results}

Eligible studies and data summary. A total of 480 studies were first identified for evaluation. Based on the criteria described in the methods, 19 publications were eligible for inclusion in the present meta-analysis $(10,12,15-31)$. The bibliographies of these 19 publications were also searched, but this did not provide further studies for inclusion. Therefore, a final total of 19 studies with 6,180 patients were included. The search process is described in Fig. 1. The anthracycline-based and/or paclitaxel regimens were the most common NAC regimens applied. Table I describes the characteristics of the eligible studies in more detail.

pCR in patients with TNBC and non-TNBC. A total of $13 \mathrm{stu}$ dies $(10,15-20,24,25,27-30)$ reported the pCR rates in patients with TNBC and non-TNBC who received NAC. There was no heterogeneity between the results of different studies $\left(\mathrm{I}^{2}=23 \%, \mathrm{P}=0.21\right)$, so the fixed-effects model was applied for data analysis. The pCR rates in the patients with TNBC were significantly higher than those in the patients with non-TNBC (OR, 3.10; 95\% CI, 2.51-3.82; Fig. 2). 
Table I. Characteristics of eligible studies.

\begin{tabular}{|c|c|c|c|c|c|}
\hline First author, year (ref.) & Study types & Total patients, $\mathrm{n}$ & Median age, years & Stages & NAC regimens ${ }^{\mathrm{a}}$ \\
\hline Bidard et al, 2008 (18) & Retrospective & 293 & 50 & I-III & FEC or FAC x(4-6) \\
\hline Chang et al, 2010 (19) & Prospective & 74 & 49 & II-III & $\mathrm{TC} \times 4$ \\
\hline Darb-Esfahani et al, 2009 (20) & Prospective & 913 & - & II-III & AT $x 4$, or AC $x 4+$ docetaxel $x 4$ \\
\hline Fisher et al, 2012 (21) & Retrospective & 385 & 50 & I-III & - \\
\hline Frasci et al, 2009 (22) & Prospective & 74 & 48 & II-III & AT + cisplatin $\times 8$ \\
\hline Medioni et al, 2011 (27) & Prospective & 74 & 50 & II-III & $\begin{array}{l}\text { Docetaxel+gemcitabine } \times 2 \text {, } \\
\text { or vinorelbine+epirubicin } \times 2\end{array}$ \\
\hline Keam et al, 2011 (23) & Prospective & 105 & - & II-III & Docetaxel or Adriamycin x 3 \\
\hline Li et al, 2011 (24) & Retrospective & 316 & 50 & I-III & $\mathrm{CAF}+$ taxanes \\
\hline Li et al, 2011 (12) & Prospective & 220 & 48 & II-III & AT x(4-6) \\
\hline Liedtke et al, 2008 (25) & Prospective & 1118 & 48 & I-III & $\begin{array}{c}\text { FAC/FEC/AC, or TFAC/TFEC, } \\
\text { or Single-agent taxane }\end{array}$ \\
\hline Masuda et al, 2011 (26) & Prospective & 163 & 50 & I-III & FEC $x 4$, or AT $x 4$ \\
\hline Ono et al, 2012 (28) & Prospective & 474 & 53 & II-III & $\mathrm{AC} / \mathrm{AT} / \mathrm{CEF}$ \\
\hline Tang et al, 2012 (29) & Retrospective & 198 & - & I-III & CEF/CMF/paclitaxel \\
\hline Wu et al, 2011 (30) & Retrospective & 249 & 47 & II-III & $\mathrm{AT} \times 4$ \\
\hline Yoo et al, 2012 (31) & Retrospective & 276 & 44 & I-III & - \\
\hline Jia et al, 2012 (17) & Retrospective & 249 & 47 & II-III & ET \\
\hline Sun et al, 2009 (15) & Prospective & 326 & 47 & II-III & CTF x4 \\
\hline Wang and Gao, 2010 (16) & Retrospective & 535 & 45 & I-III & FEC $x 4$ \\
\hline Zhou et al, 2009 (10) & Retrospective & 138 & 51 & II-III & AT x4 \\
\hline
\end{tabular}

${ }^{\mathrm{a}} \mathrm{x}$ ' indicates number of cycles (e.g. $\mathrm{x} 4$, four cycles). FEC, cyclophosphamide+epirubicin+fluorouracil; FAC, cyclophosphamide+Adriamyc in+fluorouracil; TC, docetaxel+cyclophosphamide; AT, Adriamycin+docetaxel; ET, epirubicin+docetaxel; AC, Adriamycin+docetaxel; CAF, cyclophosphamide+Adriamycin+fluorouracil; CEF, cyclophosphamide+epirubicin+fluorouracil; CTF, cyclophosphamide+docetaxel+fluorour acil; TFAC, docetaxel+FAC; TFEC, docetaxel+FEC.

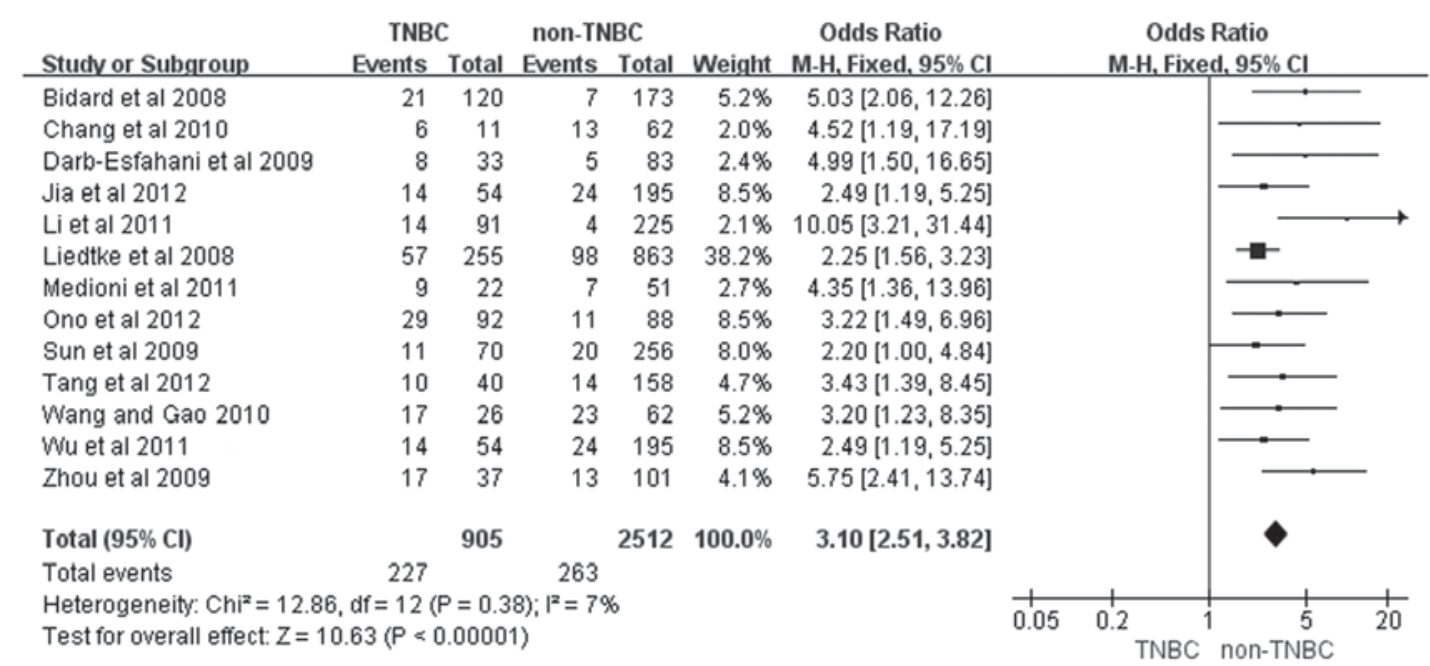

Figure 2. Forest plot: Pathological complete response rate in patients with TNBC and non-TNBC who received neoadjuvant chemotherapy. TNBC, triple-negative breast cancer; CI, confidence interval.

Survival in patients with TNBC and non-TNBC. A total of 6 studies $(10,16,17,24,25,30)$ reported the 5-year DFS rate in patients with TNBC or non-TNBC who received NAC. There was significant heterogeneity between the different research results $\left(\mathrm{I}^{2}=65 \%, \mathrm{P}=0.01\right)$, so the random-effects model was applied for data analysis. The 5-year DFS rate in the patients with TNBC was significantly lower than that in the patients with non-TNBC (54.6 vs. 70.8\%; OR, 0.53; 95\% CI, 0.34-0.81; Fig. 3).

A total of 7 studies $(10,15-17,24,25,30)$ reported the 5 -year OS rate in patients with TNBC or non-TNBC who received NAC. There was no heterogeneity between the results of the different studies $\left(\mathrm{I}^{2}=5 \%, \mathrm{P}=0.39\right)$, so the fixed-effects model was applied 


\begin{tabular}{|c|c|c|c|c|c|c|c|c|c|c|}
\hline Stucty or Subgroup & $\begin{array}{l}\text { TNBC } \\
\text { Events }\end{array}$ & Total & $\begin{array}{l}\text { non-Th } \\
\text { Events }\end{array}$ & $\begin{array}{l}\text { NBC } \\
\text { Total }\end{array}$ & Weight & $\begin{array}{c}\text { Odds Ratio } \\
\text { M-H, Random, } 95 \% \mathrm{Cl}\end{array}$ & & $\begin{array}{r}\text { Odds } \\
\text { M-H, Rando }\end{array}$ & $\begin{array}{l}\text { Ratio } \\
\text { om. } 95 \% \mathrm{Cl}\end{array}$ & \\
\hline Jia et al 2012 & 28 & 54 & 138 & 195 & $17.4 \%$ & $0.44[0.24,0.82]$ & & & & \\
\hline Li et al 2011 & 15 & 91 & 70 & 225 & $17.3 \%$ & $0.44[0.23,0.81]$ & & & & \\
\hline Liedtke et al 2008 & 156 & 225 & 604 & 863 & $23.6 \%$ & $0.97[0.70,1.33]$ & & & & \\
\hline Wang and Gao 2010 & 17 & 26 & 45 & 62 & $11.2 \%$ & $0.71[0.27,1.90]$ & & & & \\
\hline Wu et al 2011 & 28 & 54 & 138 & 195 & $17.4 \%$ & $0.44[0.24,0.82]$ & & & & \\
\hline Zhou et al 2009 & 22 & 37 & 85 & 101 & $13.2 \%$ & $0.28[0.12,0.64]$ & & & & \\
\hline Total $(95 \% \mathrm{Cl})$ & & 487 & & 1641 & $100.0 \%$ & $0.53[0.34,0.81]$ & & & & \\
\hline Total events & 266 & & 1080 & & & & & & & \\
\hline \multicolumn{7}{|c|}{$\begin{array}{l}\text { Heterogeneity: } \text { Tau }^{2}=0.17 ; \mathrm{Chi}^{2}=14.49, \mathrm{df}=5(P=0.01) ; \mathrm{I}^{2}=65 \% \\
\text { Test for overall effect: } Z=2.93(P=0.003)\end{array}$} & 0.01 & 0.1 TNBC $^{1}$ & $\begin{array}{c}10 \\
\text { non-TNBC }\end{array}$ & 100 \\
\hline
\end{tabular}

Figure 3. Forest plot: 5-year diseasae-free survival rates in patients with TNBC and non-TNBC who received neoadjuvant chemotherapy. TNBC, triple-negative breast cancer; $\mathrm{CI}$, confidence interval.

\begin{tabular}{|c|c|c|c|c|c|c|c|c|c|c|}
\hline Study or Subgroup & $\begin{array}{l}\text { TNBC } \\
\text { Events }\end{array}$ & Total & $\begin{array}{l}\text { non-TN } \\
\text { Events }\end{array}$ & $\begin{array}{l}\text { BC } \\
\text { Total }\end{array}$ & Weight & $\begin{array}{l}\text { Odds Ratio } \\
\text { M-H, Fixed, 95\% Cl }\end{array}$ & \multicolumn{4}{|c|}{$\begin{array}{c}\text { Odds Ratio } \\
\text { M-H, Fixed, } 95 \% \mathrm{Cl}\end{array}$} \\
\hline Jia et al 2012 & 32 & 54 & 157 & 195 & $13.2 \%$ & $0.35[0.18,0.67]$ & & $\because$ & & \\
\hline Li et al 2011 & 17 & 91 & 82 & 225 & $18.2 \%$ & $0.40[0.22,0.72]$ & & $\rightarrow-$ & & \\
\hline Liedtke et al 2008 & 163 & 225 & 699 & 863 & $37.8 \%$ & $0.62[0.44,0.87]$ & & $=$ & & \\
\hline Sun et al 2009 & 47 & 70 & 217 & 256 & $14.5 \%$ & $0.37[0.20,0.67]$ & & $\rightarrow$ & & \\
\hline Wang and Gao 2010 & 19 & 26 & 50 & 62 & $3.8 \%$ & $0.65[0.22,1.90]$ & & & - & \\
\hline Wu et al 2011 & 42 & 54 & 157 & 195 & $7.2 \%$ & $0.85[0.41,1.76]$ & & — & & \\
\hline Zhou et al 2009 & 28 & 37 & 85 & 101 & $5.3 \%$ & $0.59[0.23,1.47]$ & & $\rightarrow$ & t & \\
\hline Total $(95 \% \mathrm{Cl})$ & & 557 & & 1897 & $100.0 \%$ & $0.52[0.42,0.65]$ & & $\checkmark$ & & \\
\hline Total events & 348 & & 1447 & & & & & & & \\
\hline $\begin{array}{l}\text { Heterogeneity: } \mathrm{Chi}^{2}= \\
\text { Test for overall effect: }\end{array}$ & $\begin{array}{l}3.32, d f= \\
z=5.83(\end{array}$ & $\begin{array}{l}6(P= \\
0<0.0\end{array}$ & $0.39) ; 1^{2}=$ & & & & 0.01 & $0.1{ }^{0}{ }^{1}$ & $\begin{array}{c}10 \\
\text { non-TNBC }\end{array}$ & $100^{\circ}$ \\
\hline
\end{tabular}

Figure 4. Forest plot: 5-year overall survival rates in patients with TNBC and non-TNBC who received neoadjuvant chemotherapy. TNBC, triple-negative breast cancer; CI, confidence interval.

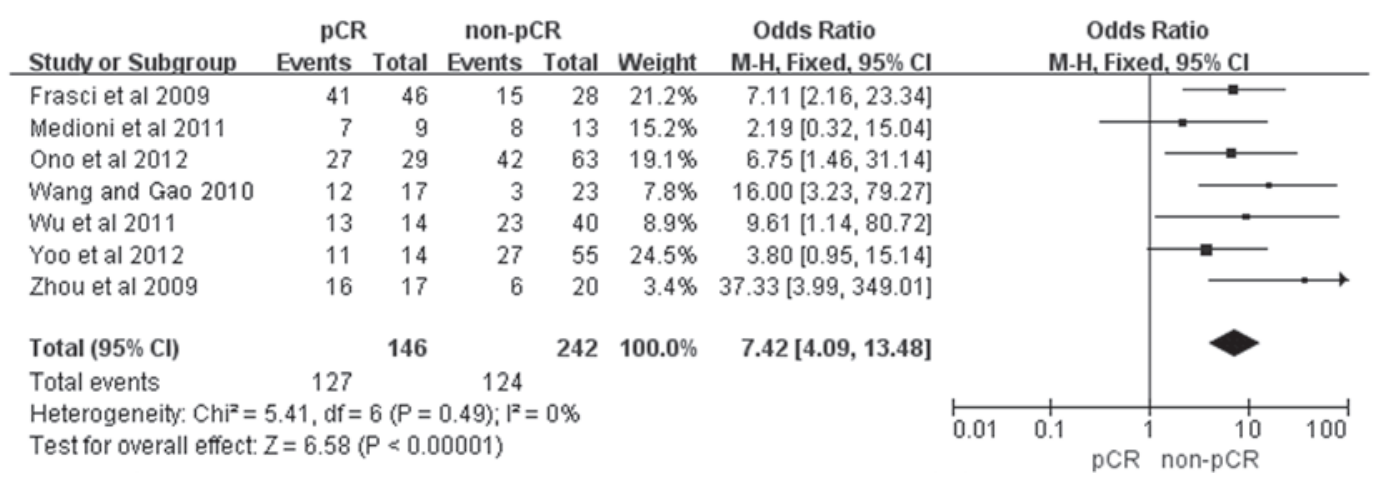

Figure 5. Forest plot: 5-year disease-free survival rates in patients with triple-negative breast cancer who received neoadjuvant chemotherapy according to the achievement of a pCR. CI, confidence interval; pCR, pathological complete response.

for data analysis. The 5-year OS rate in the patients with TNBC was significantly lower than that in the patients with non-TNBC (62.5 vs. 80.7\%; OR, 0.52; 95\% CI, 0.42-0.65; Fig. 4).

Survival rate of patients with TNBC as a function of $p C R$. For the 7 studies $(10,16,22,27,28,30,31)$ that reported the 5 -year DFS rate in the patients with TNBC who received NAC according to the achievement of a pCR, there was no heterogeneity between the results $\left(\mathrm{I}^{2}=0 \%, \mathrm{P}=0.49\right)$, therefore, the fixed-effects model was applied for data analysis. The 5-year DFS rate was significantly higher among the patients with TNBC who achieved a pCR than among those who did not achieve a pCR (OR, 7.42; 95\% CI, 4.09-13.48; Fig. 5).
For the 7 studies $(10,16,21,22,27,30,31)$ that reported the 5-year OS rate in patients with TNBC who received NAC according to the achievement of a pCR, there was also no heterogeneity between the results $\left(\mathrm{I}^{2}=0 \%, \mathrm{P}=0.59\right)$, therefore, the fixed-effects model was applied for data analysis. The 5-year OS rate was significantly higher among the patients with TNBC who achieved a pCR than among those who did not achieve a pCR (OR, 6.74; 95\% CI, 3.63-12.52; Fig. 6).

Association between molecular marker expression and pCR in patients with TNBC following NAC. A total of 6 studies $(12,18,20,23,26,28)$ reported the association between molecular marker expression and the $\mathrm{pCR}$ rate in the patients 


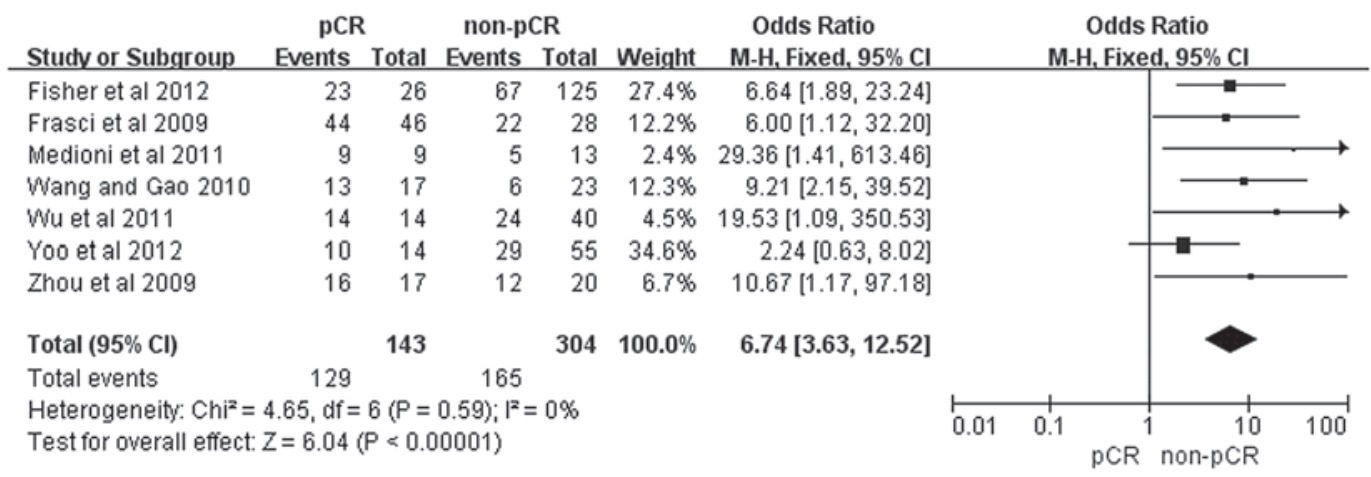

Figure 6. Forest plot: 5-year overall survival rates in patients with triple-negative breast cancer who received neoadjuvant chemotherapy according to the achievement of a pCR. CI, confidence interval; pCR, pathological complete response.

\begin{tabular}{|c|c|c|c|c|c|c|c|c|}
\hline \multirow[b]{2}{*}{ Study or Subgroup } & \multicolumn{2}{|c|}{ high Ki-67 TNBC } & \multicolumn{2}{|c|}{ Iow Ki-67 TNBC } & \multirow[b]{2}{*}{ Weight } & \multirow{2}{*}{$\begin{array}{c}\text { Odds Ratio } \\
\text { M-H, Fixed, } 95 \% \mathrm{Cl}\end{array}$} & \multirow{2}{*}{\multicolumn{2}{|c|}{$\begin{array}{c}\text { Odds Ratio } \\
\text { M-H. Fixed, } 95 \% \mathrm{Cl}\end{array}$}} \\
\hline & Events & Total & Events & Total & & & & \\
\hline Darb-Esfahani et al 2009 & 7 & 11 & 0 & 19 & $4.4 \%$ & $65.00[3.11,1359.77]$ & & \\
\hline Keam et al 2011 & 14 & 77 & 0 & 28 & $18.5 \%$ & $13.02[0.75,225.83]$ & & \\
\hline Li et al 2011 & 14 & 27 & 2 & 14 & $39.5 \%$ & $6.46[1.21,34.55]$ & & \\
\hline Masuda et al 2011 & 10 & 20 & 2 & 13 & $37.7 \%$ & $5.50[0.96,31.43]$ & & \\
\hline Total $(95 \% \mathrm{Cl})$ & & 135 & & 74 & $100.0 \%$ & $9.87[3.53,27.62]$ & & \\
\hline Total events & 45 & & 4 & & & & & \\
\hline $\begin{array}{l}\text { Heterogeneity. } \mathrm{Chi}^{2}=2.19 \\
\text { Test for overall effect: } \mathrm{Z}=4\end{array}$ & $\begin{array}{l}d f=3(P= \\
36(P<0.0\end{array}$ & 3); $1^{2}=$ & & & & & $\begin{array}{lc}0.01 & 0.1 \\
\text { high } \mathrm{Ki}-67 \mathrm{TNBC}\end{array}$ & $\begin{array}{lcc}10 & 100 \\
\text { low Ki- } 67 \text { TNBC }\end{array}$ \\
\hline
\end{tabular}

Figure 7. Forest plot: Pathological complete response as a function of the Ki-67 expression level in patients with triple-negative breast cancer who received neoadjuvant chemotherapy. CI, confidence interval.

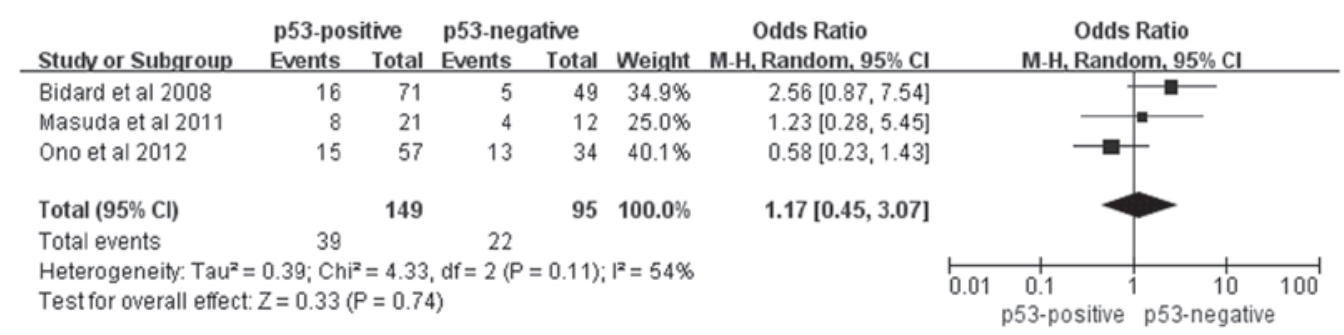

Figure 8. Forest plot: Pathological complete response as a function of the p53 expression level in patients with triple-negative breast cancer who received neoadjuvant chemotherapy. CI, confidence interval.

\begin{tabular}{|c|c|c|c|c|c|c|c|c|c|c|}
\hline Study or Subgroup & \multicolumn{2}{|c|}{ CK5/6(+) } & \multicolumn{2}{|c|}{ CK5/6(-) } & Weight & $\begin{array}{c}\text { Odds Ratio } \\
\text { M-H, Random, } 95 \% \mathrm{Cl}\end{array}$ & \multicolumn{3}{|c|}{$\begin{array}{c}\text { Odds Ratio } \\
\text { M-H, Random, } 95 \% \mathrm{Cl}\end{array}$} & \\
\hline Darb-Esfahani et al 2009 & 3 & 6 & 5 & 25 & $30.9 \%$ & $4.00[0.61,26.12]$ & & & $=$ & \\
\hline Li et al 2011 & 4 & 18 & 11 & 23 & $36.7 \%$ & $0.31[0.08,1.24]$ & & & & \\
\hline Masuda et al 2011 & 2 & 14 & 10 & 19 & $32.4 \%$ & $0.15[0.03,0.86]$ & & & & \\
\hline Total $(95 \% \mathrm{Cl})$ & & 38 & & 67 & $100.0 \%$ & $0.54[0.09,3.25]$ & & & & \\
\hline Total events & 9 & & 26 & & & & & & & \\
\hline $\begin{array}{l}\text { Heterogeneity: } \operatorname{Tau}^{2}=1.7 \\
\text { Test for overall effect: } Z=\end{array}$ & $\begin{array}{l}C h i^{2}=6.9 \\
67(P=0 .\end{array}$ & $\begin{array}{l}39, d f= \\
50)\end{array}$ & $2(P=0$. & $03) ; 1^{2}=$ & $=71 \%$ & & 0.01 & $\begin{array}{ll}0.1 & 1 \\
\text { CK5 } 5 / 6(+)\end{array}$ & $1 \frac{10}{C K 5 / 6(-)}$ & 100 \\
\hline
\end{tabular}

Figure 9. Forest plot: Pathological complete response as a function of the CK5/6 expression level in patients with triple-negative breast cancer who received neoadjuvant chemotherapy. CK, cytokeratin; CI, confidence interval.

with TNBC who received NAC. A pooled study of 4 of these studies $(12,20,23,26)$ showed that the patients with TNBC and high Ki-67 expression achieved significantly higher pCR rates than those with low Ki-67 expression (OR, 9.87; 95\% CI,3.53-27.62; Fig. 7).In addition, two pooled analyses of p53 $(18,26,28)$ and CK5/6 $(12,20,26)$ levels revealed no association between these molecules and $\mathrm{pCR}$ rate ( $\mathrm{P}>0.05$; Figs. 8 and 9$)$.
Quality evaluation. The quality of the meta-analysis was evaluated using the GRADE framework and is shown in Table II. The quality of the investigation of the 5-year DFS rate in the patients with TNBC with or without a pCR was high. The quality for the study of the 5-year DFS rate in the patients with TNBC or non-TNBC was low. The other assessments were considered to be of moderate quality. The main reason for the lower quality was 
Table II. Grading of Recommendations Assessment, Development and Evaluation framework assessment of eligible studies.

\begin{tabular}{|c|c|c|c|c|c|c|c|c|}
\hline \multicolumn{3}{|c|}{ Design } & \multicolumn{4}{|c|}{ Quality assessment } & \multirow[b]{2}{*}{$\begin{array}{c}\text { No. of eligible } \\
\text { studies }\end{array}$} & \multirow[b]{2}{*}{ Quality } \\
\hline Outcome & Experiment & Control & $\begin{array}{l}\text { Publication } \\
\text { bias }\end{array}$ & Inconsistency & Indirectness & Imprecision & & \\
\hline $\mathrm{pCR}$ & TNBC & Non-TNBC & Yes & No & No & No & 14 & Moderate \\
\hline 5-year DFS & TNBC & Non-TNBC & Yes & No & No & Yes & 6 & Low \\
\hline 5-year OS & TNBC & Non-TNBC & No & No & No & Yes & 7 & Moderate \\
\hline 5-year DFS & $\mathrm{pCR}$ & Non-pCR & No & No & No & No & 7 & High \\
\hline 5-year OS & $\mathrm{pCR}$ & Non-pCR & Yes & No & No & No & 7 & Moderate \\
\hline $\mathrm{pCR}$ & High Ki-67 & Low Ki-67 & Yes & No & No & No & 6 & Moderate \\
\hline
\end{tabular}

TNBC, triple-negative breast cancer; DFS, disease-free survival; OS, overall survival; pCR, pathological complete response.

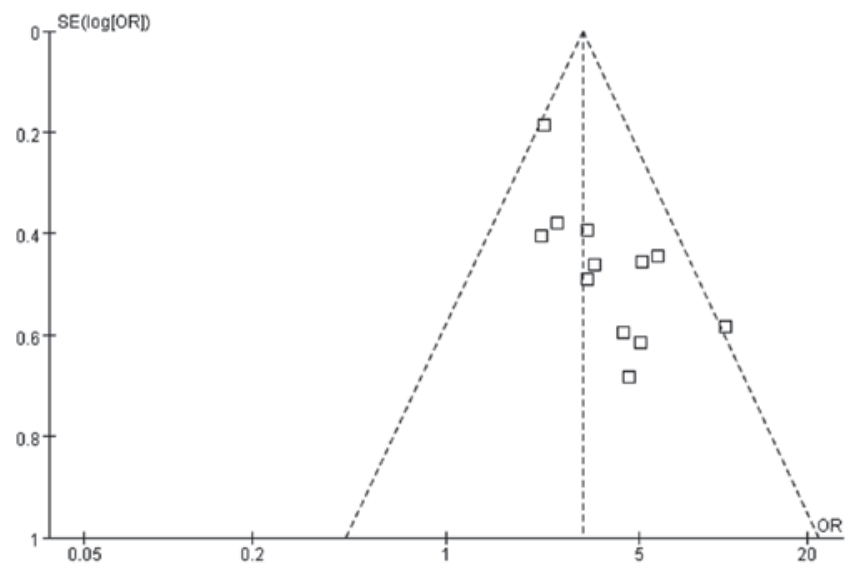

Figure 10. Funnel plot: Pathological complete response in patients with TNBC and non-TNBC who received neoadjuvant chemotherapy. TNBC, triple-negative breast cancer; OR, odds ratio; SE, standard error.

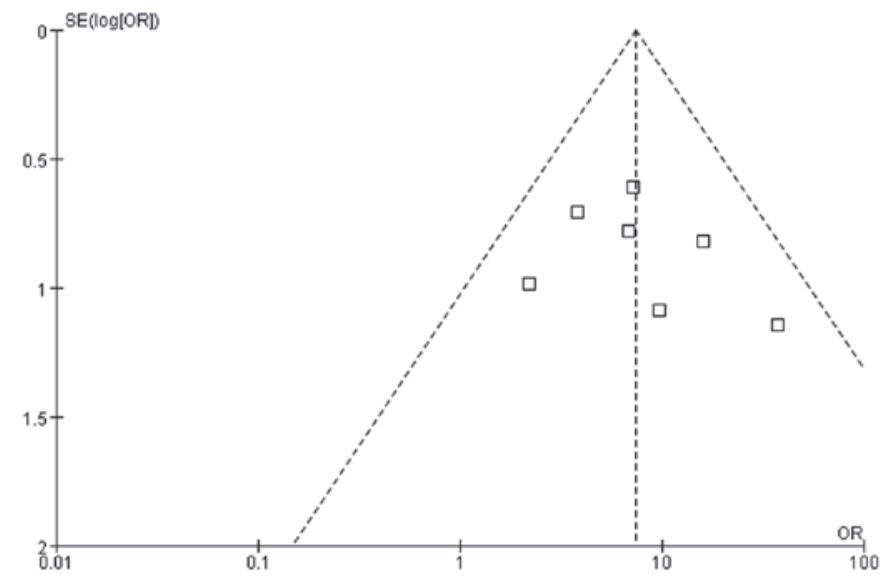

Figure 11. Inverse funnel plot: 5-year disease-free survival rates in patients with triple-negative breast cancer who received neoadjuvant chemotherapy according to the achievement of a pathological complete response. OR, odds ratio; SE, standard error.

publication bias. For example, in Fig. 10, the lower left region of the funnel plot is vacant, with points distributed throughout the remainder of funnel, suggesting a publication bias; this may be due to the difficulty in publishing studies with negative results. In Fig. 11, the points in the inverted funnel plot show homogeneous distribution on each side, suggesting no clear publication bias.

\section{Discussion}

Patients with TNBC generally have aggressive cancer with higher metastatic and lower survival rates than those with non-TNBC. However, TNBC patients generally achieve a higher pCR rate following NAC treatment compared with those individuals with 
other subtypes of breast cancer, as was confirmed by the present meta-analysis. In the current meta-analysis, the 5-year DFS and OS rates of patients with TNBC who received NAC were lower than those of patients with non-TNBC. However, a significant improvement in the 5-year DFS and OS rates was apparent in the patients with TNBC who achieved $\mathrm{pCR}$ as a result of NAC treatment, suggesting that NAC significantly improves the survival of patients with TNBC, but only for those who show a pCR to treatment.

Certain studies have suggested that different NAC regimens have a different effect on the pCR in breast cancer patients. For example, patients achieve a higher pCR rate and long-term survival rate when paclitaxel is used in anthracycline-based NAC regimens $(8,32)$. In addition, platinum-based NAC regimens also affect the survival rate (33). The NAC regimens of eligible studies in the present meta-analysis were mostly anthracycline and/or paclitaxel regimens.

TNBC can be classified as chemosensitive or chemoresistant (34), distinguished by an analysis of the tumoral expression of molecular marker genes, including EGFR, CK5/6, cyclooxygenase-2, Y-box binding protein-1, B-cell lymphoma 2, Ki-67 antigen and p53 tumor suppressor. It is generally easier to achieve a pCR in patients with chemosensitive disease, therefore, an analysis of molecular marker expression in patients with TNBC would be useful in predicting the response to NAC. The current meta-analysis showed that patients with TNBC characterized by high levels of Ki-67 antigen expression achieved a higher pCR rate than those with low-level expression, suggesting that $\mathrm{Ki}-67$ could be used as a predictor of prognosis and for the selection of patients who would derive the greatest clinical benefit from NAC. However, more studies are required to confirm the association between Ki-67 and patient prognosis. A clinical trial has reported that patients with TNBC can benefit from chemotherapy combined with molecularly targeted therapy in the form of poly-ADP ribose polymerase inhibition, and more detailed studies are underway (35).

The TNM stages of the patients included in the present meta-analysis were I-III/II-III. Therefore, it is possible that the ambiguity of the cancer stage could have introduced a bias in the data; however, the quality of these studies was considered to be mostly moderate on analysis. In addition, the overall results were reliable despite a certain degree of publication bias.

In summary, despite moderate quality and a certain degree of publication bias, a number of conclusions can be made. The survival rates in the patients with TNBC were significantly lower than those in patients with non-TNBC, but the patients with TNBC achieved a higher pCR rate in response to NAC treatment. Furthermore, the patients with TNBC who achieved a higher $\mathrm{pCR}$ rate in response to NAC treatment showed significant improvement in survival rates. Finally, high Ki-67 expression was positively correlated with a higher $\mathrm{pCR}$ rate, whereas $\mathrm{p} 53$ and CK5/6 expression did not display any prognostic function. Future multicenter randomized controlled trials would provide additional support to the current study and aid in determining whether other molecular markers can act as prognostic factors.

\section{Acknowledgements}

This meta-analysis was supported by the Programme for National Natural Science Foundation of China (grant no. 30800278) and the Doctoral Fund of Ministry of Education of China (Youth Scholars; grant no. 200804861048).

\section{References}

1. Perou CM, Sørlie T, Eisen MB, et al: Molecular portraits of human breast tumours. Nature 406: 747-752, 2000.

2. Dent R, Trudeau M, Pritchard KI, et al: Triple-negative breast cancer: clinical features and patterns of recurrence. Clin Cancer Res 13: 4429-4434, 2007.

3. Bauer KR, Brown M, Cress RD, et al: Descriptive analysis of estrogen receptor (ER)-negative, progesterone receptor (PR)-negative and HER2-negative invasive breast cancer, the so-called triple-negative phenotype: a population-based study from the California cancer Registry. Cancer 109: 1721-1728, 2007.

4. Charfare H, Limongelli S and Purushotham AD: Neoadjuvant chemotherapy in breast cancer. Br J Surg 92: 14-23, 2005.

5. Fisher B, Bryant J, Wolmark N, et al: Effect of preoperative chemotherapy on the outcome of women with operable breast cancer. J Clin Oncol 16: 2672-2685, 1998.

6. Wolmark N, Wang J, Mamounas E, et al: Preoperative chemotherapy in patients with operable breast cancer: nine-year results from National Surgical Adjuvant Breast and Bowel Project B-18. J Natl Cancer Inst Monogr: 96-102, 2001.

7. Bear HD, Anderson S, Smith RE, et al: Sequential preoperative or postoperative docetaxel added to preoperative doxorubicin plus cyclophosphamide for operable breast cancer: National Surgical Adjuvant Breast and Bowel Project Protocol B-27. J Clin Oncol 24: 2019-2027, 2006.

8. Heys SD, Sarkar T and Hutcheon AW: Primary docetaxel chemotherapy in patients with breast cancer: impact on response and survival. Breast Cancer Res Treat 90: 169-185, 2005.

9. Houssami N, Macaskill P, von Minckwitz G, Marinovich ML and Mamounas E: Meta-analysis of the association of breast cancer subtype and pathologic complete response to neoadjuvant chemotherapy. Eur J Cancer 48: 3342-3354, 2012.

10. Zhou B, Xie F, Wang S and Yang D: Response and prognosis of taxanes and anthracyclines neoadjuvant chemotherapy in patients with triple-negative breast cancer. Zhongguo Aizheng Zazhi 19: 129-132, 2009 (In Chinese).

11. Kong X, Moran MS, Zhang N, et al: Meta-analysis confirms achieving pathological complete response after neoadjuvant chemotherapy predicts favourable prognosis for breast cancer patients. Eur J Cancer 47: 2084-2090, 2011.

12. Li XR, Liu M, Zhang YJ, et al: CK5/6, EGFR, Ki-67, cyclin D1 and $\mathrm{nm} 23-\mathrm{H} 1$ protein expressions as predictors of pathological complete response to neoadjuvant chemotherapy in triple-negative breast cancer patients. Med Oncol 28 (Suppl 1): S129-S134, 2011.

13. Zhang G, Xie W, Xu L, Liu Z and Xie X: Predictors of neoadjuvant chemotherapy for triple-negative breast cancer: a meta-analysis with 723 cases. Chinese-German J Clin Oncol 12: 15-19, 2013.

14. Livasy CA, Perou CM, Karaca G, et al: Identification of a basal-like subtype of breast ductal carcinoma in situ. Hum Pathol 38: 197-204, 2007.

15. Sun ZK, Ma XT, Wu YD, et al: Response and Long-Term Effect of Patients with Triple-Negative Breast Cancer Receiving Neo-Adjuvant Anthracycline-Based Chemotherapy. Clin Oncol Cancer Res 6: 197-202, 2009.

16. Wang J and Gao R: Relationship among clinical characteristics, response and prognosis of neoadjuvant chemotherapy in patients with triple negative breast cancer. Zhong Liu Yan Jiu Yu Lin Chuang 22: 833-836, 2010 (In Chinese).

17. Jia H, Wu J, Li S, Gu R and Su F: Curative effects and prognostic evaluation on neoadjuvant chemotherapy of epirubicin combined with docetaxel for treating triple negative breast cancer. Ling Nan Xian Dai Lin Chuang Wai Ke 12: 261-265, 2012 (In Chinese).

18. Bidard FC, Matthieu MC, Chollet P, et al: p53 status and efficacy of primary anthracyclines/alkylating agent-based regimen according to breast cancer molecular classes. Ann Oncol 19: $1261-1265,2008$

19. Chang HR, Glaspy J, Allison MA, et al: Differential response of triple-negative breast cancer to a docetaxel and carboplatin-based neoadjuvant treatment. Cancer 116: 4227-4237, 2010.

20. Darb-Esfahani S, Loibl S, Müller BM, et al: Identification of biology-based breast cancer types with distinct predictive and prognostic features: role of steroid hormone and HER 2 receptor expression in patients treated with neoadjuvant anthracycline/taxane-based chemotherapy. Breast Cancer Res 11: R69, 2009. 
21. Fisher CS, Ma CX, Gillanders WE, et al: Neoadjuvant chemotherapy is associated with improved survival compared with adjuvant chemotherapy in patients with triple-negative breast cancer only after complete pathologic response. Ann Surg Oncol 19: 253-258, 2012.

22. Frasci G, Comella P, Rinaldo M, et al: Preoperative weekly cisplatin-epirubicin-paclitaxel with G-CSF support in triple-negative large operable breast cancer. Ann Oncol 20 1185-1192, 2009.

23. Keam B, Im SA, Lee KH, et al: Ki-67 can be used for further classification of triple negative breast cancer into two subtypes with different response and prognosis. Breast Cancer Res 13: R22, 2011.

24. Li J, Gonzalez-Angulo AM, Allen PK, et al: Triple-negative subtype predicts poor overall survival and high locoregional relapse in inflammatory breast cancer. Oncologist 16: 1675-1683, 2011.

25. Liedtke C, Mazouni C, Hess KR, et al: Response to neoadjuvant therapy and long-term survival in patients with triple-negative breast cancer. J Clin Oncol 26: 1275-1281, 2008

26. Masuda H, Masuda N, Kodama Y, et al: Predictive factors for the effectiveness of neoadjuvant chemotherapy and prognosis in triple-negative breast cancer patients. Cancer Chemother Pharmacol 67: 911-917, 2011.

27. Medioni J, Huchon C, Le Frere-Belda MA, et al: Neoadjuvant dose-dense gemcitabine plus docetaxel and vinorelbine plus epirubicin for operable breast cancer: improved prognosis in triple-negative tumors. Drugs R D 11: 147-157, 2011.

28. Ono M, Tsuda H, Shimizu C, et al: Tumor-infiltrating lymphocytes are correlated with response to neoadjuvant chemotherapy in triple-negative breast cancer. Breast Cancer Res Treat 132: 793-805, 2012.
29. Tang Y, Zhu L, Li Y, et al: Overexpression of epithelial growth factor receptor (EGFR) predicts better response to neo-adjuvant chemotherapy in patients with triple-negative breast cancer. J Transl Med 10 (Suppl 1): S4, 2012.

30. Wu J, Li S, Jia W and Su F: Response and prognosis of taxanes and anthracyclines neoadjuvant chemotherapy in patients with triple-negative breast cancer. J Cancer Res Clin Oncol 137: $1505-1510,2011$

31. Yoo C, Ahn JH, Jung KH, et al: Impact of immunohistochemistry-based molecular subtype on chemosensitivity and survival in patients with breast cancer following neoadjuvant chemotherapy. J Breast Cancer 15: 203-210, 2012.

32. Smith IC, Heys SD, Hutcheon AW, et al: Neoadjuvant chemotherapy in breast cancer: significantly enhanced response with docetaxel. J Clin Oncol 20: 1456-1466, 2002.

33. Sirohi B, Arnedos M,Popat S, et al: Platinum-based chemotherapy in triple-negative breast cancer. Ann Oncol 19: 1847-1852, 2008

34. De Laurentiis M, Cianniello D, Caputo R, et al: Treatment of triple negative breast cancer (TNBC): current options and future perspectives. Cancer Treat Rev 36 (Suppl 3): S80-S86, 2010.

35. O'Shaughnessy J, Osborne C, Pippen JE, et al: Iniparib plus chemotherapy in metastatic triple-negative breast cancer. $N$ Engl J Med 364: 205-214, 2011 